\title{
Some unusual discrete VLF emissions observed at a low-latitude ground station at Agra
}

\author{
B. Singh \\ Department of Physics, R. B. S. College, Bichpuri, Agra-283105, India
}

Received: 17 July 1996 / Revised: 7 February 1997 / Accepted: 17 February 1997

\begin{abstract}
A detailed analysis of the VLF emissions data obtained during occasional whistler campaigns at the low-latitude ground station Agra (geomagnetic latitude $17^{\circ} 1^{\prime} \mathrm{N}, L=1.15$ ) has yielded some unusual discrete VLF emissions of the rising type. These include (1) emissions occurring at time intervals increasing in geommetrical progression, (2) emissions occuring simultaneously in different frequency ranges and (3) emissions observed during daytime. In the present study, the observed characteristics of these emissions are described and interpreted. It is shown that the increasing time delay between different components of the emissions match closely with the propagation time delays between different hops of a whistler of dispersion $19 \mathrm{~s}^{1 / 2}$, the unusual occurrence of the emissions in two different frequency ranges approximately at the same time may possibly be linked with their generation at two different locations, and the occurrence of emissions during daytime may be due to propagation under the influence of equatorial anomaly.
\end{abstract}

\section{Introduction}

The wave-particle interactions occurring in the magnetosphere generate a variety of emissions in the ELF/VLF range. Helliwell (1965) has classified these emissions into hiss, discrete, periodic, chorus, and triggered emissions. Rycroft (1972) and Sazhin and Hayakawa (1992) have presented excellent reviews on magnetospheric chorus emissions observed in the ground stations, rockets, and satellites. Nunn and Sazhin (1991) and Trakhtengertz et al. (1996) have presented chorus models recently.

Though, the VLF emissions are basically a middleand high-latitude phenomena, there are ample evidences of their occurrence in low-latitude ground stations also. Japanese workers (Tanaka et al., 1974; Hayakawa et al., 1975; Hayakawa and Tanaka, 1977) have reported the observations of hiss-type emissions in their low latitude ground stations, whereas Indian workers (Singh et al., 1981; Khosa et al., 1983) have reported the observations of discrete-type emissions in their ground stations. The existence of intense zones of ELF/VLF emissions in the low-latitude ionosphere has been confirmed by satellite observations also (Bullough et al., 1974).

In the present study, we pick up some interesting cases of unusual discrete VLF emissions observed at our low-latitude ground station Agra during two different whistler campaign periods and interpret their unusual characteristics.

\section{Observations}

The VLF emissions are observed with the same set of equipments as that required for the observations of whistlers. This includes an antenna, pre and main amplifiers and a magnetic tape recorder. The whistler/emission data over the magnetic tapes are analysed using a digital sonograph machine.

We have conducted two major whistler campaigns at our Agra station, one in 1979 which was an independent campaign, and the other during 1989-1993 which was a coordinated campaign with three other stations, Srinagar, Varanasi, and Bhopal. During our first campaign, we used a 25 feet long horizontal antenna placed at a height of 20 feet over a 30 feet high double storey building. Using this antenna, the observations were taken during night time only from $1800 \mathrm{~h}$ to $0600 \mathrm{~h} \mathrm{LT}$. This antenna was replaced by a T-type vertical antenna during our recent whistler campaign and the observations were extended to daytime also (but in 1991 only).

\section{The unusual data}

In Fig. 1, we show the frequency - time spectrogram of the unusual discrete rising emissions which we recorded on 12 January, 1979 during the earlier whistler campaign. The interesting point in this figure is that the time 


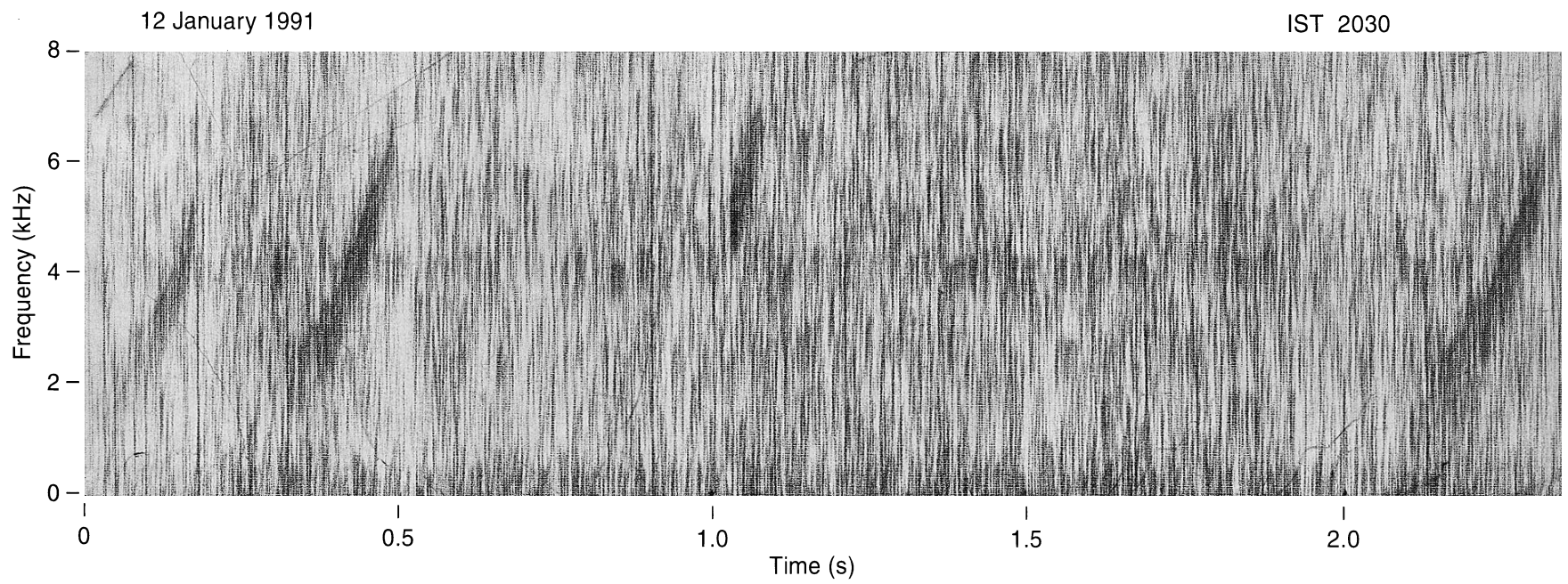

Fig. 1. Discrete emissions of the rising type recorded at Bichpuri Agra. The emissions occurred at frequency intervals approximately in geommetrical progression (reproduced from Singh et al. 1981)

intervals between the consecutive emissions are approximately in geommetrical progression. Further, the frequency ranges of the emissions increase with time except in the third emission in which case the lower cut off is at a higher frequency. Singh et al. (1981) have reported this result earlier together with other emission data obtained during the same day of observation, but they could not explain the unusual characteristics mentioned.

In Fig. 2, we show the frequency - time spectrograms of two cases of unusual emissions recorded at our station during the recent campaign period. In Fig. 2a, there are two emissions in the frequency ranges of $1.5-3.5 \mathrm{kHz}$ and 4-5 kHz that occurred almost simultaneously, and in
Fig. 2b, the rising emission was recorded during daytime, for the first time at a low-latitude ground station.

\section{Interpretation of the data}

Singh et al. (1981) have shown that the emissions of Fig. 1 were generated near the equatorial region at $L=1.2$ under cyclotron resonance mechanism and propagated to our station in nonducted mode of propagation. We interpret the unusual relationship between the time intervals of these emissions as follows; we assume that the consecutive emissions of this event
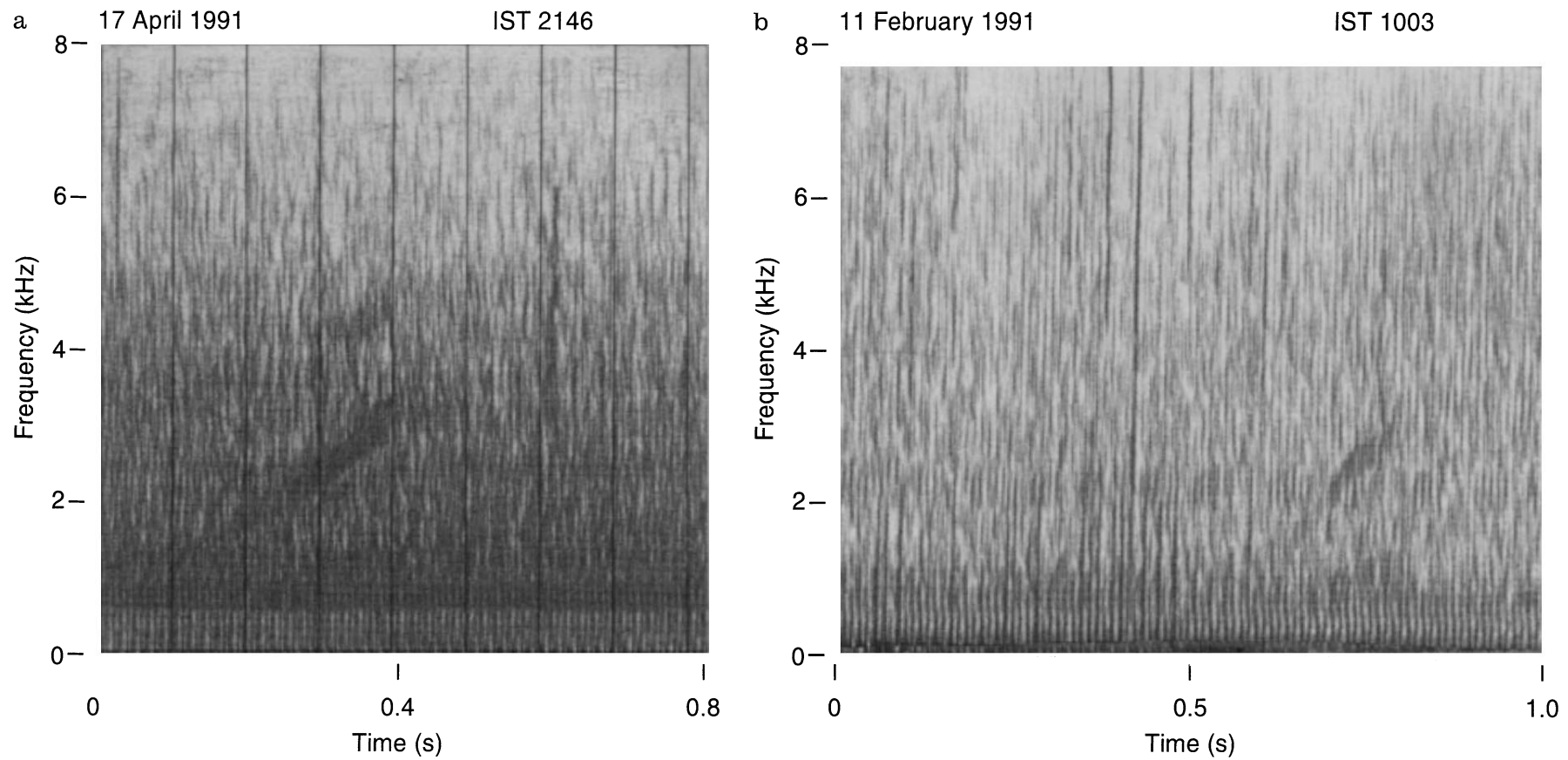

Fig. 2 a. Discrete emissions observed in two frequency ranges at almost the same time; $\mathbf{b}$ an example of rising emissions recorded during daytime 
Table 1. A comparison of time intervals between different emissions of Fig. 1 with the time delays between different hops of a whistler of dispersion $19 \mathrm{~s}^{1 / 2}$

\begin{tabular}{|c|c|c|c|c|c|}
\hline \multirow[t]{2}{*}{ S.No. } & \multicolumn{2}{|c|}{ Emission } & \multicolumn{2}{|c|}{ Whistler } & \multirow[t]{2}{*}{ Difference, $\%$} \\
\hline & Traces & Time intervalat $5 \mathrm{kHz}, \mathrm{s}$ & Hops & Time delay s & \\
\hline 1 & $1-2$ & 0.27 & $1-2$ & 0.27 & 0.0 \\
\hline 2 & $2-3$ & 0.60 & $2-4$ & 0.54 & 11.18 \\
\hline 3 & $3-4$ & 1.23 & $4-8$ & 1.08 & 12.19 \\
\hline
\end{tabular}

were generated as a result of interactions between the trapped energetic particles and the increasing hops of a magnetospherically reflected whistler. Under this assumption, we measure the time intervals between the consecutive emissions at the frequency of $5 \mathrm{kHz}$ and then match them with time delays between various hops of a whistler. We find that the calculated time intervals match closely within $12.5 \%$ with those of the $1-2,2-4$, and 4-8 hops of a whistler of dispersion $19 \mathrm{~s}^{1 / 2}$. The detailed results are presented in Table 1 . This provides a possible explanation to the observed characteristics. The increasing frequency ranges of the emissions are explained in terms of shifting upward in $L$ of the generation region for each emissions and increasing lengths of the interaction regions.

The possibility that the occurrence of these emissions was just a coincidence does not seem to be likely because Singh et al. (1981) has reported other cases also in which two emissions occurred one after the other during the same night of observation, although, the event shown in Fig. 1 was a single case of this type.

We have examined various possibilities for the occurrence of the emissions shown in Fig. 2a which include (1) that they were caused by instrument, (2) they were generated due to second order cyclotron resonances (3) they were caused by HF heating of the lower ionosphere. However, these possibilities are ruled out

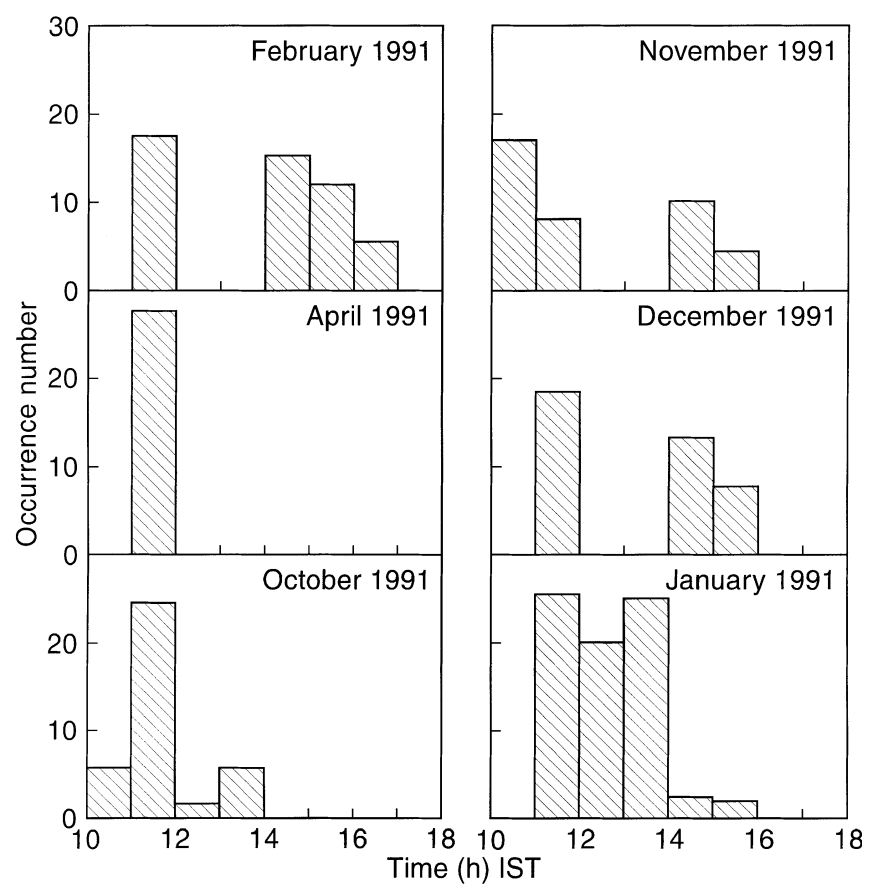

Fig. 3. Diurnal variation of occurrence number of daytime emissions because such emissions were also recorded at Varanasi (Singh, 1991), the second order cyclotron resonance requires energetic electrons of $\sim 50 \mathrm{MeV}$ which are not available at $L=1.2$ (Kartz, 1966), and radiations from HF heating peaks at frequency near $2 \mathrm{kHz}$ which are not observable on the ground if the receiver is away from the source (Stubbe, 1994). Thus, the only possibility left is that these emissions are generated at two slightly different locations in the magnetosphere.

The daytime emissions, one of which is shown in Fig. 2b, have been observed for the first time at a lowlatitude station. Normally, such waves are not transmitted to the ground due to heavy absorption in the lower ionosphere and large wave normal angles associated with them. However, since Japanese workers have observed large number of whistlers during daytime and interpreted their propagation to ground in terms of the effect of the equatorial anomaly (Tanaka and Hayakawa, 1985), it is possible that the emissions recorded by us also propagated to our station under the influence of the anomaly. The diurnal variation of their occurrence number shown in Fig. 3 clearly indicates their association with the developing phase of the anomaly.

Acknowledgement. The author is grateful to the Department of Science and Technology, Government of India, New Delhi, for providing financial assistance for this work.

The Editor in chief thanks S. S. Sazhin and another referee for their help in evaluating this paper.

\section{References}

Bullough, K., A. R. W. Hughes, and T. R. Kaiser, Space craft studies of VLF emissions, in Magnetospheric Physics, Ed. B. M. McCormac, 231-240, Reidel, Dordrecht, Holland, 1974.

Hayakawa, M. and Y. Tanaka, ELF emissions observed at Moshiri, Nature (London), 270, 703-705, 1977.

Hayakawa, M., Y. Tanaka, and J. Ohtsu, Satellite and ground observations of magnetospheric VLF emissions associated with severe magnetic storm of 25-27 May, 1967, J. Geophys. Res., 80, 86-92, 1975.

Helliwell, R. A. Whistlers and related ionospheric phenomena, Stanford University Press, Stanford, Cal., 206-207, 1965.

Kartz, L., Electron and proton observations, in Radiations trapped in the earth's magnetic field, Ed. B. M. McCormac, 129-154, Reidel, Dordrecht, Holland, 1966.

Khosa, P. N., Lalmani, and M. M. Ahmed, Discrete chorus emissions recorded at Varanasi, J. Geophys., 54, 76, 1983.

Nunn, D. and S. S. Sazhin, On the generation mechanism of hiss triggered emissions, Ann. Geophysicae, 9, 603-610, 1991.

Rycroft, M. J., VLF emissions in the magnetosphere, Radio Sci., 7, 811-830, 1972.

Sazhin, S. S. and M. Hayakawa, Magnetospheric chorus emissions: a review, Planet. Space Sci., 40, 681-697, 1992.

Singh, B., R. Prakash, and H. Singh, Discrete chorus emissions observed at a low latitude station, Nature (London), 290, 37-39, 1981. 
Singh, H., R. Prakash, and B. Singh, A study of whistlers observed at Agra, Indian J. Radio Space Phys., 9, 130-133, 1980.

Singh, R. P., Personal Communication, Banaras Hindu University, Varanasi, October, 1991.

Stubbe, P., Personal Communication, Max-Plank Institute for Aeronomie, Germany, July, 1994.

Tanaka, Y., and M. Hayakawa, On the propagation of daytime whistlers at low latitudes, J. Geophys. Res., 90, 3457-3464, 1985.
Tanaka, Y., M. Hayakawa, and J. Ohtsu, VLF hiss observed at a low latitude ground station and its relation to drifting ring current electrons, J. Ionos. Space Res. (Japan), 28, 168-172, 1974.

Trakhtengerts, V. Y., M. J. Rycroft, and A. G. Demekhov, Interrelation of noise-like and discrete ELF/VLF emissions generated by cyclotron interactions, J. Geophys. Res., 101, 13293-13301, 1996. 